\title{
Influence of inflammation on parasitism and area of experimental amoebic liver abscess: an immunohistochemical and morphometric study
}

\author{
Cássia Abadia Xavier Costa ${ }^{1,2^{*}}$, Thaisa Helena Silva Fonseca ${ }^{3}$, Fabrício Marcus Silva Oliveira ${ }^{1,2}$, \\ Joseph Fabiano Guimaraes Santos ${ }^{4}$, Maria Aparecida Gomes ${ }^{3}$, Marcelo Vidigal Caliari ${ }^{2^{*}}$
}

\begin{abstract}
The influence of inflammation on the number of trophozoites and on the murine amoebic liver abscess area following infection with Entamoeba histolytica and E. dispar was evaluated. Immunohistochemistry and digital morphometry were used to identify and quantify the trophozoites, neutrophils, macrophages, and lesions. Positive correlation was observed between the number of trophozoites and inflammatory cells. A significant decrease in parasitism and inflammation in groups treated with dexamethasone was observed. The scarceness or absence of trophozoites in the treated groups suggest the importance of the inflammatory response in the production of amoebic hepatic abscesses in spite of the inherent virulence of the parasite being decisive in the establishment of the lesion.
\end{abstract}

\section{Findings}

Amoebiasis is a disease caused by the protozoan Entamoeba histolytica through oral infection by cysts followed by colonization or tissue invasion by trophozoites in the large intestine. Annually, about 50 million people are infected, causing approximately 100,000 deaths [1]. After the colonic mucosa is damaged, the trophozoites may reach the bloodstream and liver, producing the amoebic hepatic abscess, which is the most common extra-intestinal form of amoebiasis [2]. The typical amoebic lesion is characterized by a liquefactive necrosis zone with edges consisting of cellular debris and polymorph-histiocitary inflammatory infiltrate. Such necrosis is produced by trophozoite derivatives, such as amoebapores (polypeptides capable of forming pores in the hosts' cells), cysteine proteinases (enzymes that cleave collagen, elastin, fibrinogen, and laminin), and galactose/ $\mathrm{N}$-acetylgalactosamine lectin (Gal/GalNac) (a CD59 like

\footnotetext{
* Correspondence: cassiaaxc@pop.com.br; caliari@icb.ufmg.br 'Programa de Pós-Graduação em Patologia, Instituto de Ciências Biológicas da Universidade Federal de Minas Gerais. Av. Antônio Carlos 6627, Belo Horizonte, Minas Gerais, Brasil

${ }^{2}$ Departamento de Patologia Geral, Instituto de Ciências Biológicas da Universidade Federal de Minas Gerais. Av. Antônio Carlos 6627, Belo Horizonte, Minas Gerais, Brasil

Full list of author information is available at the end of the article
}

molecule capable of inhibiting the C5b-9 complex of the complement, inducing apoptosis and stimulating the production of IL- $1 \alpha$, IL-1 $\beta$, and IL-8).

Controversial results have been published about the role of inflammation on the pathogenesis of amoebic necrosis. The inflammatory infiltrate in amoebiasis is very discrete when compared to intense necrosis, suggesting that the inflammatory cells are destroyed, releasing their enzymatic content into the hepatic parenchyma [2]. Radiationinduced leucopenia in hamsters followed by trophozoites inoculation led to the reduction of inflammatory processes, hepatic necrosis, and parasitism [3]. On the other hand, mice infected with $E$. histolytica and previously treated with anti-neutrophil monoclonal antibodies developed liver necrosis with the same dimensions as those of the control group, and those lesions were probably produced by products secreted by the amoeba itself [4].

Considering the doubts that still persist about amoebic pathogenesis, we assessed the influence of inflammation in necrosis and in hepatic parasitism in mice inoculated with $E$. histolytica. For comparison purposes, we used the non-pathogenic species $E$. dispar, which can produce experimental lesions similar to those of E. histolytica and that has contributed to the understanding of the pathogenesis of amoebiasis [5,6]. Trophozoites from 
both strains, neutrophils, and macrophages were identified by immunohistochemistry and quantified by digital morphometry.

All procedures were conducted according to principles established by the Ethics Committee on Animal Experimentation of the UFMG. Ten Swiss mice were intrahepatically inoculated with 250,000 trophozoites of the EGG strain of E. histolytica or MCR strain of E. dispar, previously identified by zymodeme and PCR $[7,8]$. Half of the animals were previously treated with two doses $(0.5 \mathrm{mg} / \mathrm{Kg})$ of dexamethasone, subcutaneously, 24 and $01 \mathrm{~h}$ before inoculation (Groups EGG-DEX and MCRDEX) and, the other half received only buffered saline (Groups EGG and MCR).

The animals were euthanized on the second day of infection, three slices of the left lobe of the liver were collected, fixed in buffered formaldehyde $10 \%$-pH 7.2, and scanned by a Sony MVC-CD400/CD250 digital camera for the morphometry of the amoebic necrosis. After dehydration, clearing and paraffin embedding of the slices, $4 \mu \mathrm{m}$ thick cuts were obtained and stained with Haematoxylin and Eosin (H\&E).

Sections from the slices were washed in phosphate buffered saline (PBS)-pH 7.2, incubated in a $\mathrm{H}_{2} \mathrm{O}_{2} 30 \mathrm{vv}-$ $3.5 \%$ solution and goat serum. In order to identify neutrophils and macrophages, the sections were incubated with a Target-Retrieval (Dako-USA) solution at $100^{\circ} \mathrm{C}$; incubated with anti-macrophage or anti-neutrophil monoclonal serum 1:50 (Hycult Biotechnology b.v.Netherlands). In order to identify the trophozoites, the sections were incubated with anti-E. histolytica or antiE. dispar polyclonal serum 1:1000 (Laboratórios de Amebiases e Protozooses/ICB/UFMG). All sections were incubated with biotinylated IgG and streptavidin conjugated with peroxidase 1:200 (Zymed Laboratories Inc., USA). In all reactions, the color was detected using a $0.05 \%$ diaminobenzidine solution in $\mathrm{H}_{2} \mathrm{O}_{2}-40 \mathrm{vv}-0.2 \%$; the sections were counterstained with Harris's haematoxylin. As a negative control, the primary antiserum was replaced by PBS in some sections (Figure 1). Positive controls consisted of sections of mouse skin inflamed by intradermal injection of carrageenan, and trophozoite-rich liver sections. Using Carl Zeiss-KS300 Software (Oberkochen, Germany), the necrotic areas of the liver slices were assessed.

Using a JVC-TK 1270 microcamera (JVC, Japan) and a $40 \times$ objective lens, 30 random images of immunohistochemical reactions against trophozoites were scanned, covering a total of $1.6 \times 10^{6} \mathrm{~mm}^{2}$ analyzed hepatic parenchyma for the counting of trophozoites by that software. The macrophages and neutrophils were quantified on the edges of the necrosis using the same methodology. The distribution of continuous variables was assessed by the Shapiro-Wilk test, and this indicated normal distribution in all cases. Thus, the Student t test was used for comparison between groups, and differences with $\mathrm{p}<0.05$ were considered statistically significant. Data were expressed as mean \pm standard deviation for parametric variables. The Pearson $r$ was used for correlation analysis.

To exclude the possibility of amebicidal activity of dexamethasone, the growth inhibition of E. histolytica and $E$. dispar cultures associated with the drug was assessed. For this, 240,000 trophozoites were grown in $6 \mathrm{~mL}$ of medium at $37^{\circ} \mathrm{C}$ and associated with dexamethasone in concentrations ranging of 40 to $800 \mathrm{mg} / \mathrm{mL}$ for 48 hours. The tests were done in triplicate and repeated one time. There was no interference of drugs on the growth and development of cultures even in higher concentrations.

Hepatic amoebic lesions were detected in all animals inoculated with E. histolytica or E. dispar. Within 48 hours of infection, the lesion was macroscopically visible in the left lobe; it had a yellowish-white color and was well defined.

Under the light microscope it was possible to observe the central area of liquefactive necrosis whose border consisted of cell debris, a discrete inflammatory infiltrate, and trophozoites. Polymorph-histiocitary inflammatory foci associated with cell debris and trophozoites were found in the control animals, as opposed to the animals treated with dexamethasone (Figure 2).

The mean and the standard deviation of the hepatic necrosis area in animals inoculated with E. histolytica of groups EGG and EGG-DEX were $2.95 \pm 1.91 \mathrm{~mm}^{2}$ and $1.32 \pm 0.44 \mathrm{~mm}^{2}$, respectively. In animals inoculated with $E$. dispar of groups MCR and MCR-DEX, they were $4.63 \pm 2.96 \mathrm{~mm}^{2}$ and $2.62 \pm 1.71 \mathrm{~mm}^{2}$, respectively. Although the mean area of necrosis proved to be arithmetically smaller in animals from both groups treated with dexamethasone, this difference between treatment groups was not significant by the Student's t-test $(p>0.05)$. The reduction of inflammation was associated with a small decrease in the necrotic area showing that necrosis in the untreated groups was mainly due to amoebic products. The secretion of amoebapores, cystein-proteinases, and Gal/GalNac lectin by the trophozoites also results in the destruction of neutrophils and the consequent liberation of their toxic products, which may play an important role in the amplification of amoebic lesions [2].

Immunohistochemistry revealed the presence of trophozoites mainly on the edges of the central zone of necrosis, as well as inside it, in the hepatic sinusoids, and in inflammatory foci. The quantitative analysis showed a significant decrease in the number of trophozoites and of inflammatory foci in the animals in groups EGG-DEX and MCR-DEX (Figures 3 and 4) (p < 0.05). In groups EGG and MCR, immunohistochemistry 


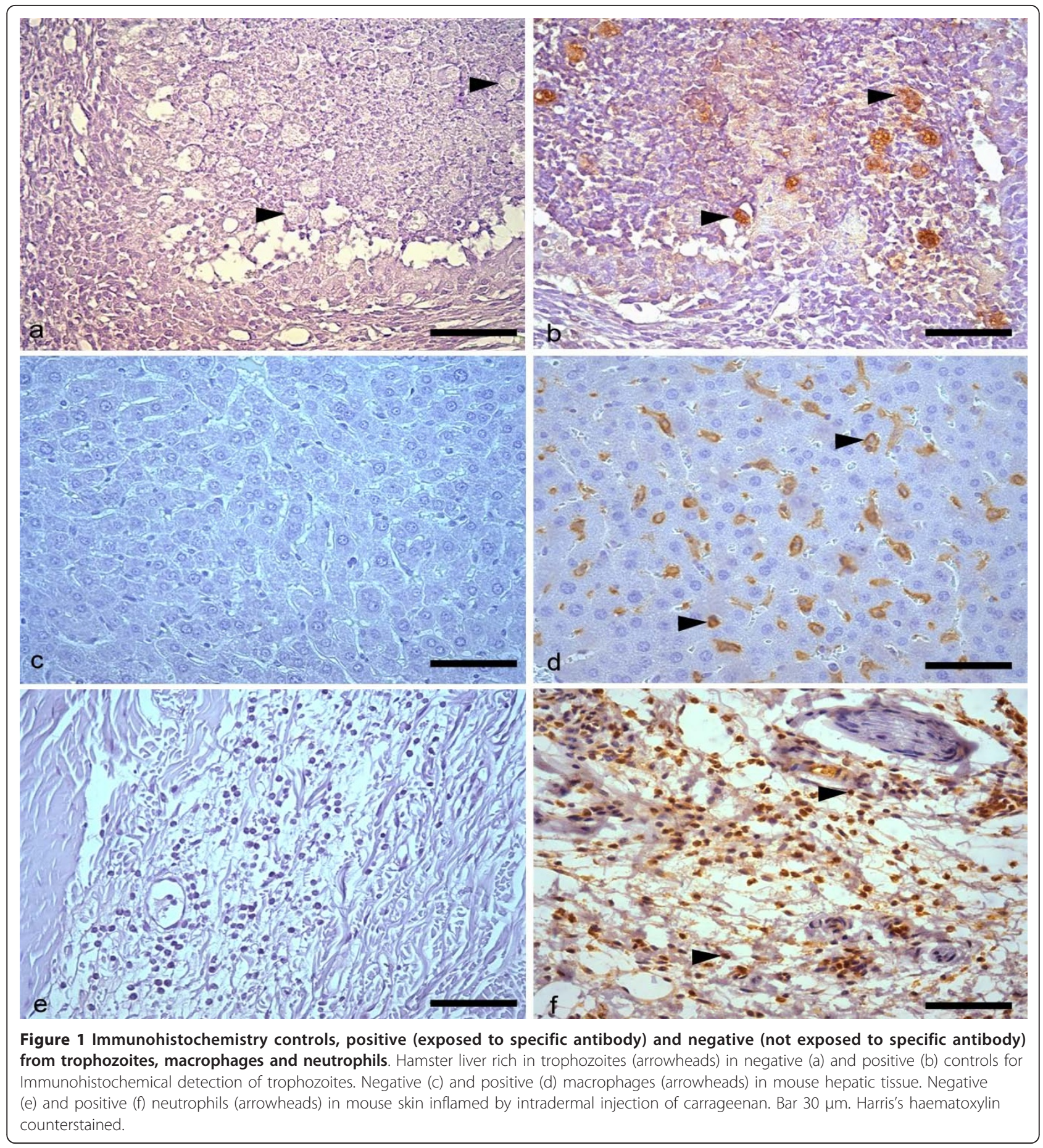

showed a predominance of macrophages in the inflammatory infiltrate, not only on the edges of the necrosis zone but also in the inflammatory foci (Figure 5), whereas the neutrophils were found in small quantities in the same areas (Figure 6). In vitro studies showed that macrophages activated after stimulation via IFNgamma showed greater resistance and ability to destroy trophozoites through NO production [9]. When stimulated with amoebic proteins, macrophages isolated from hepatic abscesses in the initial stages of infection produced large concentrations of TNF-a [10]. The need for macrophage activation suggests a secretory Th1 response of IFN-gamma, IL-2, and TNF-alpha. On the other hand, in experimental amoebic colitis the same intensity of parasitism and inflammation was observed in animals deficient in IL-2, IFN-gamma, and NO [11]. 

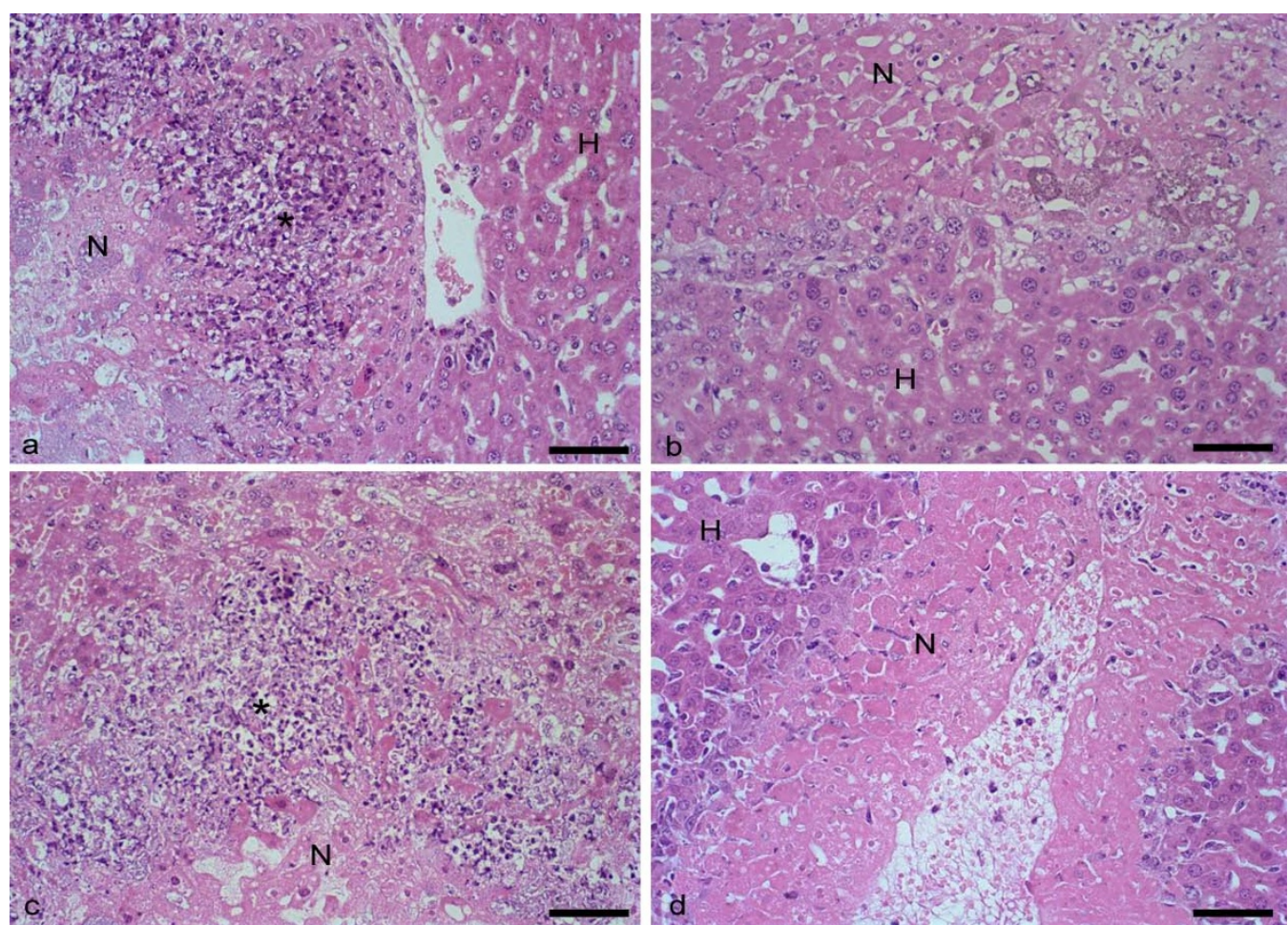

Figure 2 Mouse liver inoculated with E. histolytica and E. dispar treated and untreated with dexamethasone. Hepatic necrosis (N) induced in mice of the EGG group (a) and the MCR group (c). Debris and scarse inflammatory infiltrate $\left(^{*}\right)$. Non-necrotic hepatic parenchyma (H). EGG-DEX (b) and MCR-DEX (d) groups. Bar $50 \mu \mathrm{m}$. H\&E.
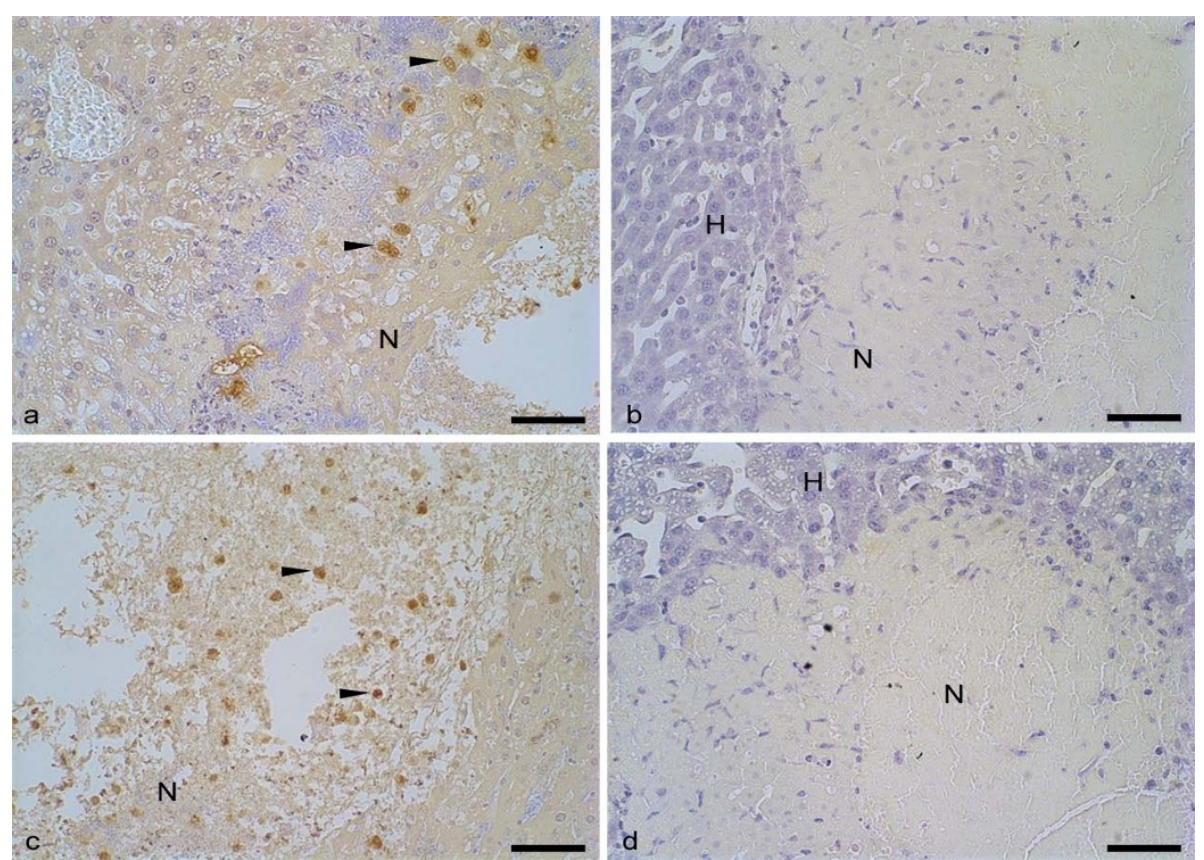

Figure $3 \mathrm{Immunohistochemical} \mathrm{reaction} \mathrm{for} \mathrm{trophozoites} \mathrm{in} \mathrm{the} \mathrm{liver} \mathrm{of} \mathrm{mice} \mathrm{inoculated} \mathrm{with} E$. histolytica or E. dispar treated and untreated with dexamethasone. Hepatic necrosis $(\mathrm{N})$ induced in mice of the EGG group (a) and the MCR group (c). Trophozoites of E. histolytica and E. dispar immunostained within the hepatic necrosis (arrowheads). Absence of trophozoites in the hepatic necrosis ( $\mathrm{N}$ ) in the groups EGG-DEX (b) and MCR-DEX (d). Non-necrotic hepatic parenchyma (H). Bar $50 \mu \mathrm{m}$. Harris's haematoxylin counterstained. 


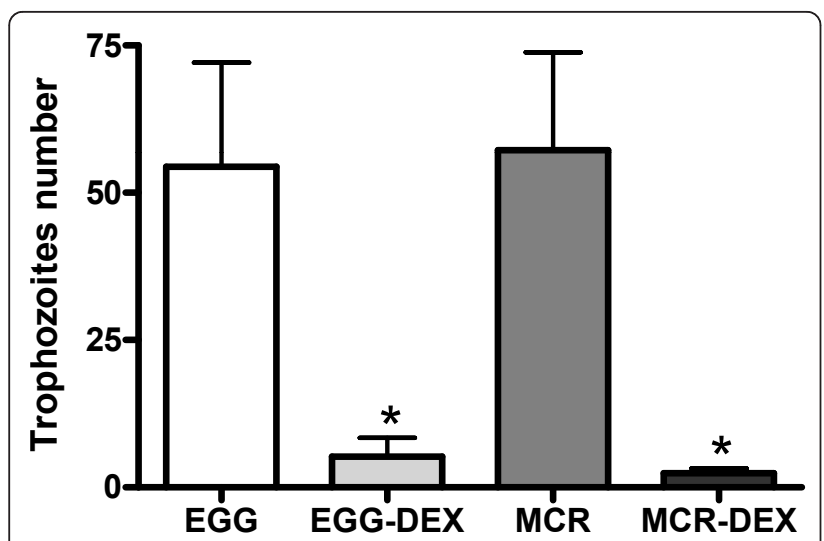

Figure 4 Number of trophozoites in the hepatic parenchyma of mice inoculated with E. histolytica (EGG) or E. dispar (MCR), treated or not with dexamethasone. ${ }^{*} t=2,73 ; p<0.05$.

The quantitative analysis showed a significant decrease of macrophages on the edges of the necrosis zone of the animals in the MCR-DEX group ( $<0.05)$. The mean and the standard deviation of the number of macrophages in animals inoculated with $E$. dispar from groups
MCR and MCR-DEX were $23 \pm 8.91$ and $5.8 \pm 5.67$, respectively. A positive correlation was observed between the fall in the number of trophozoites and the reduction in the number of macrophages in the necrotic zone $(r=0.6217 ; p=0.0017)$ and the reduction in the number of neutrophils in the hepatic parenchyma $(\mathrm{r}=$ $0.3939 ; \mathrm{p}=0.04)$. In groups EGG $(12.4 \pm 3.4)$ and EGG$\operatorname{DEX}(9.2 \pm 8.2)$ there was no significant reduction in the number of macrophages at the edge of the lesion.

As expected, the treatment with dexamethasone reduced the inflammatory infiltrate, but, paradoxically, the number of trophozoites and necrotic area were smaller. Such results suggest that the inflammation normally observed during the amoebic pathogenesis favors the multiplication of trophozoites and, consequently, leads to an increase in the dimensions of the amoebic abscess area. It is possible that leukocytes and/or their products stimulate the transcription of amoebic toxic substances, amplifying the lesion. Additionally, a study performed with trophozoites marked with radioactivity showed that less resistant trophozoites were destroyed by inflammation, favoring the proliferation of more virulent

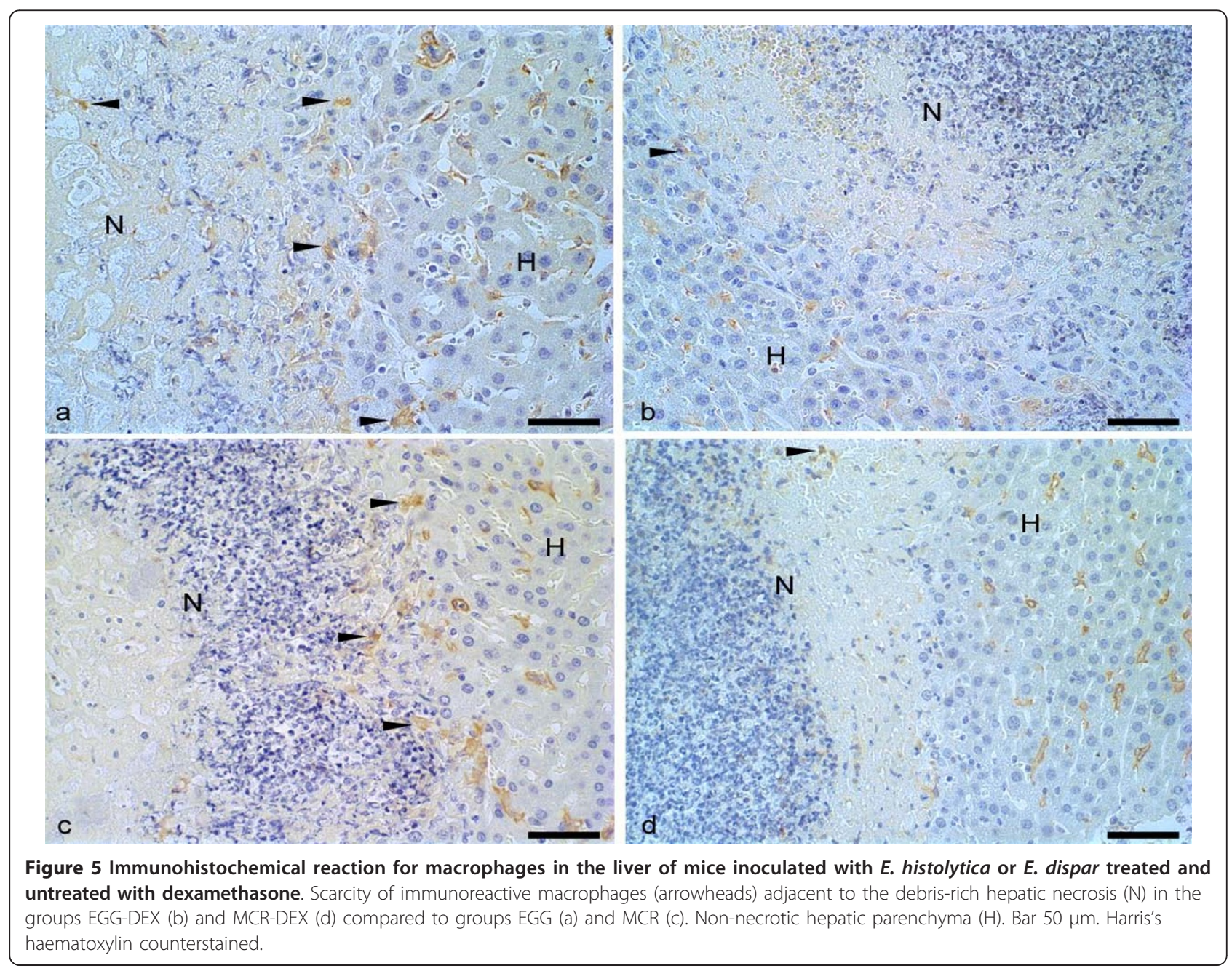




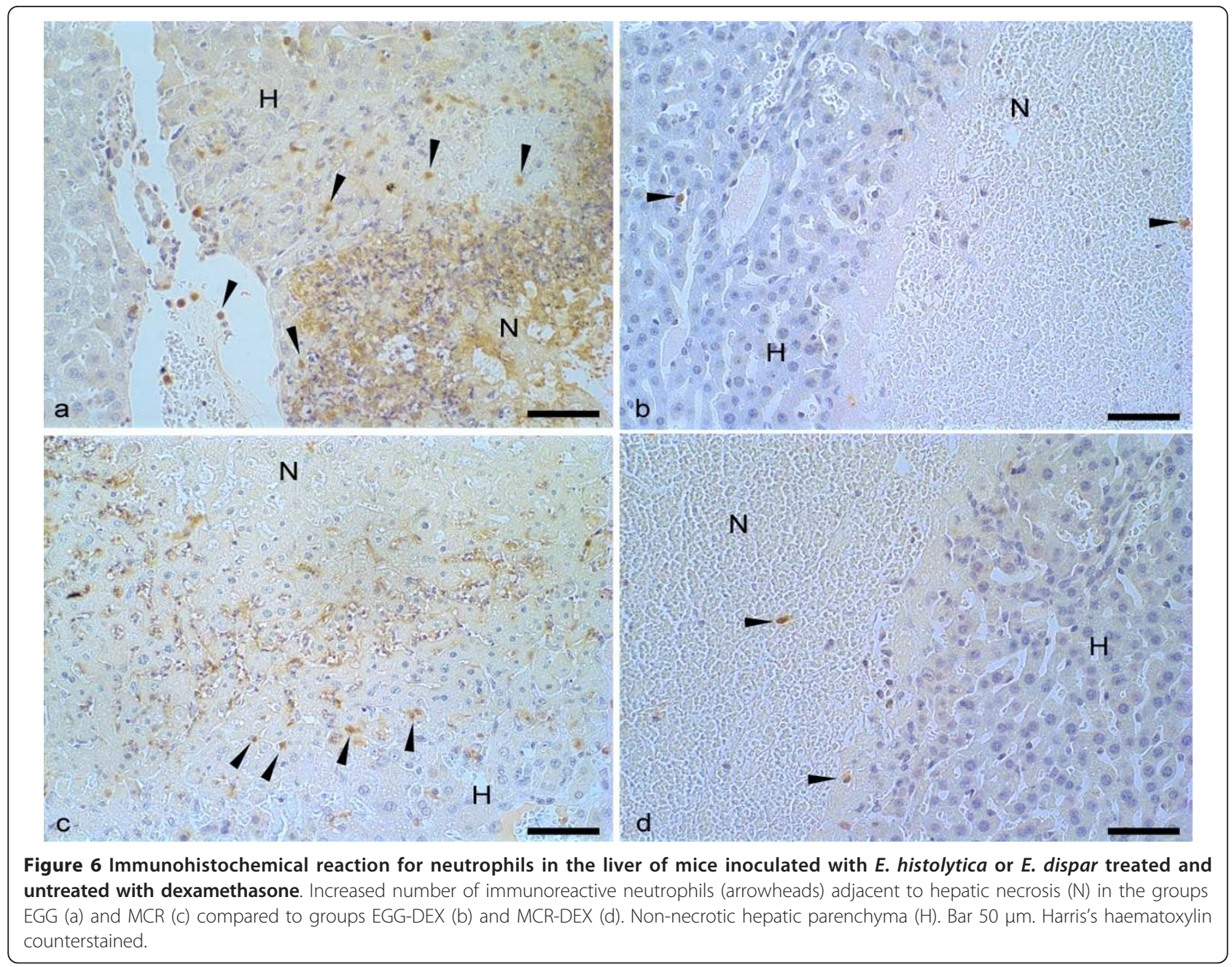

parasites [12]. This probable selection of parasites by the inflammatory process has also been indicated by our studies with the experimental model of amoebic liver abscess in hamster $[13,14]$. In animals inoculated with either E. histolytica or E. dispar small numbers of trophozoites were found in the liver parenchyma after 12 hours of infection. However, within 24 hours of infection a significant increase of trophozoites was observed in mice infected with both species of amoebae (7 and 130 times in E. histolytica and E. dispar, respectively), showing that, although both produce experimental lesions, their mechanisms of adaptation to the host are different. Perhaps the lowest number of trophozoites observed in E. histolytica infection would be related to its higher virulence. E. histolytica produces greater amount and variety of proteolytic substances, leading not only to the destruction of host tissue but also of amoebae. No significant reduction observed in the number of macrophages at the edge of the lesion in the EGG group leads us to speculate that these cells are not major factors involved in virulence selection on E. histolytica.
The visualization of residual trophozoites with positive immunohistochemical staining for immunoglobulins and complement, suggests that these elements may be involved in the selection of more resistant parasites [14]. Genotype differences from E. histolytica and E. dispar in stool- and liver abscess-derived samples from the same patients $[15,16]$ corroborate the selection hypothesis.

In spite of the earlier published controversial results about the importance of the inflammatory response in the production of amoebic hepatic abscesses, our results emphasise the crucial role of inflammation in pathogenesis and establishment of the lesion.

\section{Acknowledgements}

This work was supported by FAPEMIG (Fundação de Amparo à Pesquisa do Estado de Minas Gerais) and CNPq (Conselho Nacional de Desenvolvimento Científico e Tecnológico). The authors are grateful to João da Costa Viana, Jacqueline Vanessa C.C. de Moura and Gislene Arlindo da Silva for technical assistance.

\section{Author details}

'Programa de Pós-Graduação em Patologia, Instituto de Ciências Biológicas da Universidade Federal de Minas Gerais. Av. Antônio Carlos 6627, Belo 
Horizonte, Minas Gerais, Brasil. ${ }^{2}$ Departamento de Patologia Geral, Instituto de Ciências Biológicas da Universidade Federal de Minas Gerais. Av. Antônio Carlos 6627, Belo Horizonte, Minas Gerais, Brasil. ${ }^{3}$ Departamento de Parasitologia, Instituto de Ciências Biológicas da Universidade Federal de Minas Gerais. Av. Antônio Carlos 6627, Belo Horizonte, Minas Gerais, Brasil. ${ }^{4}$ Hospital Governador Israel Pinheiro - IPSEMG, Belo Horizonte, Minas Gerais, Brasil.

\section{Authors' contributions}

CCAX, GMA and CMV conceived the study. CCAX, GMA and FTHS by rearing the amoebas, made the inocula, liver inoculation of hamsters and in vitro tests. CCAX, OFMS and CMV made the necropsy of mice, histopathological procedures and morphometry analysis. CCAX, OFMS and FTHS made the immunohistochemical assays. SJFG made the statistical analysis. All authors participated in the study design, analysis of the results, drafted the manuscript and have given final approval of the version to be published.

\section{Competing interests}

The authors declare that they have no competing interests.

Received: 27 October 2010 Accepted: 28 February 2011

Published: 28 February 2011

\section{References}

1. Petri WA Jr, Haque R, Lyerly D, Vines RR: Estimating the impact of amebiasis on health. Parasitol Today 2000, 16:320-321.

2. Tsutsumi V, Martinez-Palomo A: Inflammatory reaction in experimental hepatic amebiasis an ultrastructural study. Am J Pathol 1988, 103:112-119.

3. Olivos-García A, Nequiz-Avendaño M, Tello E, Martínez RD, GonzálezCanto A, López-Vancell R, García de León MC, Montfort I, Pérez-Tamayo R: Inflammation, complement, ischemia and amebic survival in acute experimental amoebic liver abscess in hamsters. Exp Mol Pathol 2004, 77:66-71.

4. Velazquez C, Shibayama-Salas M, Aguirre-Garcia J, Tsutsumi V, Calderon J: Role of neutrophils in innate resistance to Entamoeba histolytica liver infection in mice. Parasite Immunol 1998, 20:255-262.

5. Costa CAX, Brito KNO, Gomes MA, Caliari MV: Histopathological and immunohistochemical study of the hepatic lesions experimentally induced by Entamoeba dispar. Eur I Histochem 2010, 54:170-174.

6. Shibayama M, Dolabella SS, Silva EF, Tsutsumi V: A Brazilian species of Entamoeba dispar (ADO) produces amoebic liver abscess in hamsters. Ann Hepatol 2007, 6:117-118.

7. Gomes MA, Pesquero JB, Furst C, Valle PR, Pesquero JL, Silva EF: An improved method to distinguish Entamoeba histolytica and Entamoeba dispar. Parasitology 1999, 119:359-362.

8. Martinez AM, Gomes MA, Viana JC, Romanha AJ, Silva EF: Isoenzyme profile as parameter to differentiate pathogenic strains of Entamoeba histolytica in Brazil. Rev Inst Med Trop São Paulo 1996, 38:407-412.

9. Lin JY, Chadee K: Macrophage cytotoxicity against Entamoeba histolytica trophozoites is mediated by nitric oxide from L-arginine. J Immunol 1992, 48:3999-4005

10. Wang W, Keller K, Chadee K: Modulation of tumor necrosis factor production by macrophages in Entamoeba histolytica infection. Infect Immun 1992, 60:3169-3174.

11. Houpt ER, Glembocki DJ, Obrig TG, Moskaluk CA, Lockhart LA, Wright RL, Seaner RM, Keepers TR, Petri WAJr: The mouse model of amebic colitis reveals mouse strain susceptibility to infection and exacerbation of disease by CD4+ T cells. J Immunol 2002, 169:4496-4503.

12. Rigothier MC, Khun H, Tavares $P$, Cardona A, Huerre M, Guillén N: Fate of Entamoeba histolytica during establishment of amoebic liver abscess analyzed by quantitative radioimaging and histology. Infect Immun 2002, 70:3208-3215.

13. Costa CAX, Brito KNO, Gomes MA, Caliari MV: Morphometric study of the hepatic lesions experimentally induced in hamsters by Entamoeba dispar and E. histolytica. Parasite 2007, 14:329-334.

14. Costa CAX, Nunes AC, Ferreira AJ, Gomes MA, Caliari MV: Entamoeba histolytica and $E$. dispar trophozoites in the liver of hamsters: in vivo binding of antibodies and complement. Parasit Vectors 2010, 3:1-10.

15. Ali IK, Solaymani-Mohammadi S, Akhter J, Roy S, Gorrini C, Calderaro A, Parker SK, Haque R, Petri WA Jr, Clark CG: Tissue Invasion by Entamoeba histolytica: Evidence of Genetic Selection and/or DNA Reorganization Events in Organ Tropism. PLoS Negl Trop Dis 2008, 2:1-6.

16. Ximénez C, Cerritos R, Rojas L, Dolabella S, Morán P, Shibayama M, González E, Valadez A, Hernández E, Valenzuela O, Limón A, Partida O, Silva EF: Human amebiasis: breaking the paradigm? Int I Environ Res Public Health 2010, 7:1105-1120.

doi:10.1186/1756-3305-4-27

Cite this article as: Costa et al:: Influence of inflammation on parasitism and area of experimental amoebic liver abscess: an immunohistochemical and morphometric study. Parasites \& Vectors 2011 $4: 27$.

\section{Submit your next manuscript to BioMed Central and take full advantage of:}

- Convenient online submission

- Thorough peer review

- No space constraints or color figure charges

- Immediate publication on acceptance

- Inclusion in PubMed, CAS, Scopus and Google Scholar

- Research which is freely available for redistribution

Submit your manuscript at www.biomedcentral.com/submit
Biomed Central 